\title{
TRANSIENT ANALYSIS OF A SINGLE SERVER QUEUEING SYSTEM WITH INFINITE BUFFER
}

\author{
Huixia Huo, Houbao Xu*, Zhuoqian Chen and Thet Thet Win
}

\begin{abstract}
As a typical single server queueing system, computer integrated manufacturing system (CIMS) has been widely used in the field of intelligent manufacturing. However, how to derive its instantaneous index is still an important issue. This paper investigates the transient behavior of the CIMS with spectral method. By constructing an asymptotic system and analyzing the spectral distribution, we derive the explicit transient solution of the asymptotic system. Trotter-Kato theorem is used to prove that the transient solution of the CIMS is just the limitation of explicit transient solution of the asymptotic system. At the end of the paper, numerical examples are shown to illustrate the effectiveness of the proposed approximation.
\end{abstract}

Mathematics Subject Classification. 90B22, 60K25.

Received June 8, 2020. Accepted October 13, 2020.

\section{INTRODUCTION}

Computer integrated manufacturing system (CIMS) is a new technology manufacturing system formed by combining computer technology with production. As a typical single server tandem queueing system, CIMS became a hot spot in the development of manufacturing industry in the early 1980s [22], and has been widely used in industry 4.0 with the theme of digitization, smart factories or Internet of Things $[35,36]$.

Single server tandem queueing system with finite buffer is commonly seen in practical systems, due to space or process constraints. Shu [34] obtained a work flow balance theorem based on system reliability theory and gave some indexes of system reliability. Sericola [31] derived a procedure for the computation of the stationary buffer content and the stationary overflow probability. $\mathrm{Xu}$ and $\mathrm{Hu}$ [39] used the Trotter-Kato theorem to analyze the approximation of a dynamic system consisting of two machines separated by a finite storage buffer. $\mathrm{Wu}$ and Zhao [37] studied the properties of tandem queues with a finite buffer capacity and non-overlapping service times subject to time-based preemptive breakdowns. Cruz et al. [9] improved the Multi-objective performance of general finite single-server queueing networks by using a mixed methodology. Afroun et al. [1] used Q-matrix method to analyze and evaluate the performance of $M / M / 1 / N$ queueing models with multiple vacations, Bernoulli feedback, balking, reneging and retention of the impatient customers, and the possibility of a server breakdown and repair. The inverse Laplace transform method and the generation function method are two common methods to solve the transient behavior of the finite buffer. Braband [3] presented transient

Keywords. CIMS, asymptotic system, spectral distribution, Trotter-Kato theorem, transient behavior.

Department of Mathematics and Statistics, Beijing Institute of Technology, Beijing 100081, P.R. China.

*Corresponding author: xuhoubao@bit.edu.cn 
solutions for a mathematical model of statistical multiplexing by inverse Laplace transform method. In Sharma et al. [33], the transient analysis of a single server Markovian queueing model having customers impatience with threshold is performed by using probability generating function technique. In addition, Sericola [30] deduced the transient solution of the flow field model based on the recursive relationship.

Single server tandem queueing system with infinite buffer has been extensively analyzed in several papers. Fleming and Simon [10] illustrated the method of generating closed-form analytic approximations for the sojourn time distribution of the $\mathrm{M} / \mathrm{M} / 1$ queue with Bernoulli feedback, the $\mathrm{M} / \mathrm{M} / 1$ processor sharing queue, a priority queue with feedback and the $\mathrm{M} / \mathrm{E}(\mathrm{k}) / 1$ queue. Golovko et al. [13] studied the queueing system with infinite buffer, single server and exponential service, and proved the existence and uniqueness of the stationary queuing system model and the stability of the non-stationary queueing system model. Ghosh and Park [12] addressed a rate control problem associated with a single server tandem Markovian queueing system and considered an infinite horizon cost minimization problem. Chaudhry et al. [6] analyzed an infinite-buffer single-server queue with renewal input and Markovian service process. Zhang et al. [40] proposed a rate iteration method embedded with a generalized extension method to model an open universal queueing network with blocking and feedback. Pradhan and Gupta [28] considered an infinite-buffer batch-service queue with Markovian arrival process and obtained a bivariate vector generating function. However, the transient behavior with infinite buffer is rarely discussed. Some references focus on studying the steady-state solution with infinite buffer $[4,7,29]$. Barbot and Sericola [2] gave an infinite-capacity buffer receiving fluid and obtained the joint stationary distribution of the buffer level by generating function. Using semigroup theory, Gupur and $\mathrm{Li}$ [14] proved that 0 is a simple eigenvalue of the system operator, Liu et al. [24] derived the steady-state solution of the system, Gupur et al. [15] and Huang [16] proved that the transient solution of the CIMS, as a typical single server tandem queueing system, is converges asymptotically to its steady-state solution.

Though the steady-state analysis provides some important information about the CIMS, the steady-state solution does not provide enough information about system performance. Transient analysis especially explicit transient solution is of great value for understanding transient behavior [11,18]. For the transient analysis of M/M/1 queueing system, Krinik [19] presented a Taylor series method for determining the transient probabilities of the classical single server queueing System, Leguesdron et al. [21] proposed a new method based on normalization technique and generation function to solve transient probability of M/M/1 queueing system, Sericola et al. [32] obtained the transient solution of a fluid queue driven by an $\mathrm{M} / \mathrm{M} / 1$ queue in terms of a modified Bessel function of the first kind by employing continued fraction methodology. However, in the transient analysis of the system, in addition to obtain the transient solution, another notable problem is the rate at which the transient solution converges to the steady-state solution. As an effective method to solve such problems, spectral distribution can not only obtain the transient solution based on the matrix decomposition, but also derive the convergence rate of the transient solution by estimating the range of eigenvalues. On the other hand, as a useful tool for studying the convergence of numerical approximation, the Trotter-Kato theorem is a projection method, which can flexibly cover various approximation schemes for infinite dimensional systems [17]. So in this paper, spectral distribution and Trotter-Kato theorem are used to investigate the explicit transient behavior of the CIMS with infinite buffer.

The rest of the paper is organized as follows: Section 2 describes the CIMS and transforms the system into an abstract Cauchy problem. It is difficult to obtain the explicit transient solution of the CIMS. To solve such problem, we construct an asymptotic system and obtain the explicit transient solution of such system in Section 3. Section 4 proves that the solution of the CIMS is the limitation of transient solution of the asymptotic system. Moreover, the convergence of transient solution of the CIMS is presented. The numerical examples of the proposed approximation are shown in Section 5. Section 6 concludes the paper.

\section{MODEL DESCRIPTION}

In this section, we will describe the CIMS and transform the system into an abstract Cauchy problem by choosing a suitable state space and defining operator. 


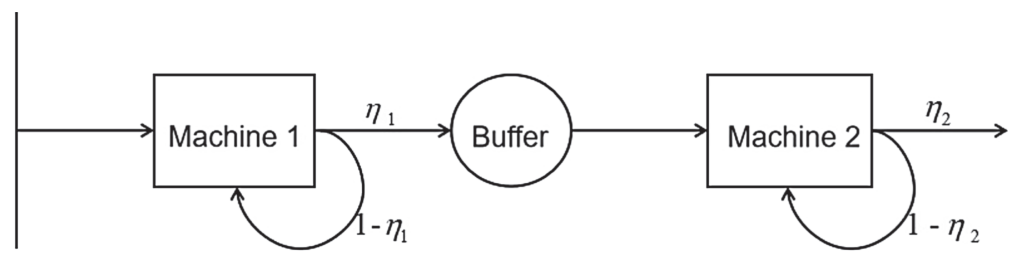

Figure 1. Behavior of the CIMS.

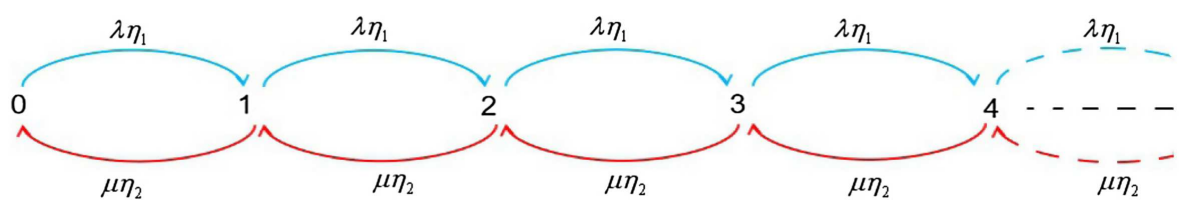

FiguRE 2. State transition diagram of the CIMS.

To better understand the CIMS, we briefly introduce the concepts of Tandem queueing system and Bernoulli feedback. Tandem queueing system is a basic structure of queueing networks in which all stations are dependent. It can be divided into a single server tandem queueing system and multiple servers tandem queueing system. Bernoulli feedback means that after each service, the workpiece will leave with a certain probability, and immediately feedback to wait for the next service with a certain probability, where the sum of these two probabilities is equal to 1.

Consider the CIMS consisting of two machines separated by a buffer just like shown in Figure 1. Workpieces enter machine 1 from outside of the system. After being processed by machine 1, a workpiece will directly go to the buffer with probability $\eta_{1}$, or it will be reprocessed by machine 1 with probability $1-\eta_{1}$. A workpiece will enter machine 2 from the buffer. After being operated on machine 2, a workpiece will exit the system with probability $\eta_{2}$, otherwise it will be reprocessed by machine 2 with probability $1-\eta_{2}$. Therefore, the CIMS can be regarded as a single server tandem queueing system with infinite buffer and Bernoulli feedbacks.

In this paper, we assume that machine 1 and machine 2 execute these workpieces one by one and the number of workpieces in the system can be infinity. The number of workpieces is the sum of workpieces in the both machines and in the buffer. The processing rates of machine 1 and machine 2 are $\lambda$ and $\mu(\lambda>0, \mu>0)$ respectively. It is reasonable to assume that $\frac{\lambda \eta_{1}}{\mu \eta_{2}}<1$, which conforms to the actual background [38].

The fluid flow model for buffer behavior analysis, which can be regarded as a generalization of the birth and death process model, has been discussed by $[8,25]$. Let $p_{0}(t)$ denotes the probability that machine 1 is processing a workpiece at time $t$ and there is no workpiece in the buffer or machine 2 . Let $p_{i}(t)(i=1,2,3, \ldots)$ denote the probability that at time $t$ both machines are busy and there are $i-1$ workpieces in the buffer. Then the state transition process of the CIMS can be considered as an M/M/1 queueing system, as shown in Figure 2.

As a typical single server queueing system, the CIMS can be governed by a group of equations by probabilistic arguments [23]:

$$
\left\{\begin{array}{l}
\frac{\mathrm{d} p_{0}(t)}{\mathrm{d} t}=-\lambda \eta_{1} p_{0}(t)+\mu \eta_{2} p_{1}(t) \\
\frac{\mathrm{d} p_{n}(t)}{\mathrm{d} t}=\lambda \eta_{1} p_{n-1}(t)-\left(\lambda \eta_{1}+\mu \eta_{2}\right) p_{n}(t)+\mu \eta_{2} p_{n+1}(t), \quad n \geq 1
\end{array}\right.
$$

with initial value:

$$
\vec{p}(0)=(1,0,0, \ldots)^{T}
$$


Let the state space $X=l^{1}=\left\{\vec{p}=\left(p_{0}, p_{1}, p_{2}, \ldots\right)^{T}\left|\sum_{n=0}^{\infty}\right| p_{n} \mid<\infty\right\}$ and define operator $A: X \rightarrow X$ as

$$
A=\left(\begin{array}{cccccc}
-\lambda \eta_{1} & \mu \eta_{2} & & & & \\
\lambda \eta_{1} & -\left(\lambda \eta_{1}+\mu \eta_{2}\right) & \mu \eta_{2} & & & \\
& \lambda \eta_{1} & -\left(\lambda \eta_{1}+\mu \eta_{2}\right) & \mu \eta_{2} & & \\
& & \ldots & \ldots & \ldots & \\
& & & \lambda \eta_{1}-\left(\lambda \eta_{1}+\mu \eta_{2}\right) \mu \eta_{2} & \ldots
\end{array}\right)
$$

The domain of $A$ is $\mathrm{D}(A)=X$. Therefore, the system (2.1) and (2.2) can be expressed as an Abstract Cauchy Problem in $X$ :

$$
\left\{\begin{array}{l}
\frac{\mathrm{d} \vec{p}(t)}{\mathrm{d} t}=A \vec{p}(t) \\
\vec{p}(0)=(1,0,0, \ldots)^{T}
\end{array}\right.
$$

where $\vec{p}(t)=\left(p_{0}(t), p_{1}(t), p_{2}(t), \ldots\right)^{T}$. The steady-state solution of the system is

$$
\vec{p}^{\star}=\left(\frac{\mu \eta_{2}-\lambda \eta_{1}}{\mu \eta_{2}}, \frac{\lambda \eta_{1}}{\mu \eta_{2}} \frac{\mu \eta_{2}-\lambda \eta_{1}}{\mu \eta_{2}},\left(\frac{\lambda \eta_{1}}{\mu \eta_{2}}\right)^{2} \frac{\mu \eta_{2}-\lambda \eta_{1}}{\mu \eta_{2}},\left(\frac{\lambda \eta_{1}}{\mu \eta_{2}}\right)^{3} \frac{\mu \eta_{2}-\lambda \eta_{1}}{\mu \eta_{2}}, \ldots\right)^{T}
$$

which derived by Liu et al. [24].

\section{Asymptotic System}

In order to study the transient behavior of the CIMS (2.4), we construct an asymptotic system as follows,

$$
\left\{\begin{array}{l}
\frac{\mathrm{d} \vec{p}^{(n)}(t)}{\mathrm{d} t}=A^{(n)} \vec{p}^{(n)}(t) \\
\vec{p}^{(n)}(0)=(1,0,0, \ldots, 0)^{T}
\end{array}\right.
$$

where $A^{(n)}(n=0,1,2, \ldots)$ is a matrix with dimensions $(n+1) \times(n+1)$,

$$
A^{(n)}=\left(\begin{array}{cccccc}
-\lambda \eta_{1} & \mu \eta_{2} & & & \\
\lambda \eta_{1} & -\left(\lambda \eta_{1}+\mu \eta_{2}\right) & \mu \eta_{2} & & \\
& \lambda \eta_{1} & -\left(\lambda \eta_{1}+\mu \eta_{2}\right) & \mu \eta_{2} & & \\
& & \cdots & \cdots & \ldots & \\
& & & \lambda \eta_{1}-\left(\lambda \eta_{1}+\mu \eta_{2}\right) & \mu \eta_{2} \\
& & & & \lambda \eta_{1} & -\mu \eta_{2}
\end{array}\right)
$$

Matrix $A^{(n)}$ in (3.2) is obviously a linear bounded operator, it can generate uniformly continuous semigroup $T^{(n)}(t)[27]$. The transient solution of the asymptotic system (3.1) is

$$
\vec{p}^{(n)}(t)=T^{(n)}(t) \vec{p}^{(n)}(0)=\exp \left(t A^{(n)}\right) \vec{p}^{(n)}(0)
$$

where $\vec{p}^{(n)}(t)=\left(p_{0}^{(n)}(t), p_{1}^{(n)}(t), p_{2}^{(n)}(t), \ldots, p_{n}^{(n)}(t)\right)^{T}$.

\subsection{The eigenvalue distribution of $A^{(n)}$}

In this subsection, in order to obtain the explicit transient solution of the asymptotic system (3.1), we will discuss the eigenvalue distribution of the asymptotic system operator $A^{(n)}$ for fixed $n$. 
Characteristic polynomials of $A^{(n)}$ is $\phi_{n}(\xi)=\left|\xi I-A^{(n)}\right|$, that is

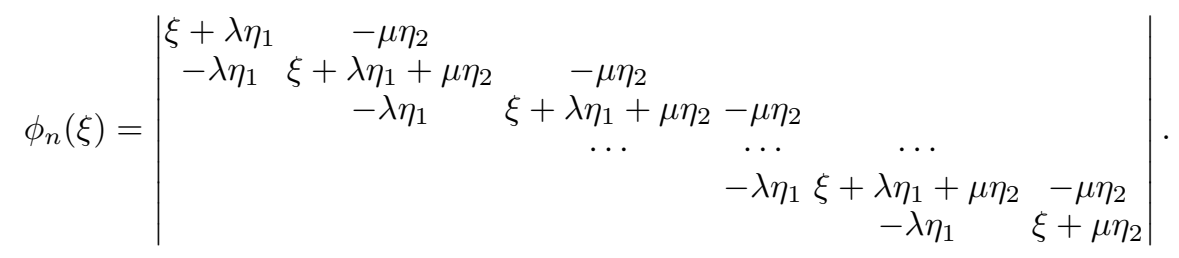

By expanding the determinant (3.4), we can obtain the recurrence relation

$$
\phi_{n}(\xi)-\left(\xi+\lambda \eta_{1}+\mu \eta_{2}\right) \phi_{n-1}(\xi)+\lambda \mu \eta_{1} \eta_{2} \phi_{n-2}(\xi)=0
$$

which is valid for $n \geq 1$ if we define $\phi_{-1}(\xi)=0, \phi_{0}(\xi)=\xi$.

For simplicity, we define the roots of $\phi_{n}(\xi)=0$ by $\alpha_{0}^{(n)}, \alpha_{1}^{(n)}, \ldots, \alpha_{n}^{(n)}$. Then we have the following theorem.

Theorem 3.1. $\alpha_{r}^{(n)}(r=0,1,2, \ldots, n)$ is the simple eigenvalue of $A^{(n)}$ and

$$
0=\alpha_{0}^{(n)}=\alpha_{0}^{(n-1)}>\alpha_{1}^{(n)}>\alpha_{1}^{(n-1)}>\cdots>\alpha_{n-1}^{(n)}>\alpha_{n-1}^{(n-1)}>\alpha_{n}^{(n)} .
$$

Proof. We observe that

$$
\left\{\begin{array}{l}
\phi_{-1}(\xi)=0 \\
\phi_{0}(\xi)=\xi \\
\phi_{1}(\xi)=\xi\left(\xi+\lambda \eta_{1}+\mu \eta_{2}\right) \\
\phi_{n}(\xi)-\left(\xi+\lambda \eta_{1}+\mu \eta_{2}\right) \phi_{n-1}(\xi)+\lambda \mu \eta_{1} \eta_{2} \phi_{n-2}(\xi)=0(n \geq 2)
\end{array}\right.
$$

implies that for any given number $\xi>0$, we have $\phi_{k}(\xi)>0$.

Since $\phi_{0}(\xi)=\xi$, the unique root of $\phi_{0}(\xi)=0$ is $\alpha_{0}^{(0)}=0$.

Let $\phi_{1}(\xi)=\xi\left(\xi+\lambda \eta_{1}+\mu \eta_{2}\right)=0, \phi_{2}(\xi)=\left(\xi+\lambda \eta_{1}+\mu \eta_{2}\right) \phi_{1}(\xi)-\lambda \mu \eta_{1} \eta_{2} \phi_{0}(\xi)=0$ and $\phi_{3}(\xi)=\left(\xi+\lambda \eta_{1}+\right.$ $\left.\mu \eta_{2}\right) \phi_{2}(\xi)-\lambda \mu \eta_{1} \eta_{2} \phi_{1}(\xi)$, we obtain

$$
\begin{aligned}
& \alpha_{0}^{(1)}=0, \quad \alpha_{1}^{(1)}=-\left(\lambda \eta_{1}+\mu \eta_{2}\right)<0, \\
& \alpha_{0}^{(2)}=0, \quad \alpha_{1}^{(2)}=-\left(\lambda \eta_{1}+\mu \eta_{2}\right)+\sqrt{\lambda \mu \eta_{1} \eta_{2}}<0, \quad \alpha_{2}^{(2)}=-\left(\lambda \eta_{1}+\mu \eta_{2}\right)-\sqrt{\lambda \mu \eta_{1} \eta_{2}}<0, \\
& \alpha_{0}^{(3)}=0, \quad \alpha_{1}^{(3)}=-\left(\lambda \eta_{1}+\mu \eta_{2}\right)+2 \sqrt{\lambda \mu \eta_{1} \eta_{2}}>\alpha_{1}^{(2)}, \\
& \alpha_{2}^{(2)}<\alpha_{2}^{(3)}=-\left(\lambda \eta_{1}+\mu \eta_{2}\right)<\alpha_{1}^{(2)}, \quad \alpha_{3}^{(3)}=-\left(\lambda \eta_{1}+\mu \eta_{2}\right)-2 \sqrt{\lambda \mu \eta_{1} \eta_{2}}<\alpha_{2}^{(2)} .
\end{aligned}
$$

Substitute $\alpha_{i}^{(1)}(i=0,1)$ into $\phi_{2}(\xi)$ and $\alpha_{i}^{(2)}(i=0,1,2)$ into $\phi_{3}(\xi)$, we have

$$
\begin{aligned}
& \phi_{2}\left(\alpha_{0}^{(1)}\right)=0, \quad \phi_{2}\left(\alpha_{1}^{(1)}\right)=\lambda \mu \eta_{1} \eta_{2}\left(\lambda \eta_{1}+\mu \eta_{2}\right)>0 . \\
& \phi_{3}\left(\alpha_{0}^{(2)}\right)=0, \quad \phi_{3}\left(\alpha_{1}^{(2)}\right)>0, \quad \phi_{3}\left(\alpha_{2}^{(2)}\right)<0 .
\end{aligned}
$$

By repeating the above procedure, suppose $\operatorname{sgn} \phi_{n}\left(\alpha_{r}^{(n-1)}\right)=(-1)^{r+1}$, the roots of $\phi_{n}(\xi)=0$ can be described as Figure 3,

The roots of $\phi_{n}(\xi)=0$ are

$$
\alpha_{0}^{(n)}=0, \quad \alpha_{r}^{(n)}=-\left(\lambda \eta_{1}+\mu \eta_{2}\right)+2 \sqrt{\lambda \mu \eta_{1} \eta_{2}} \cos \frac{r \pi}{n+1}(r=1,2, \ldots, n),
$$




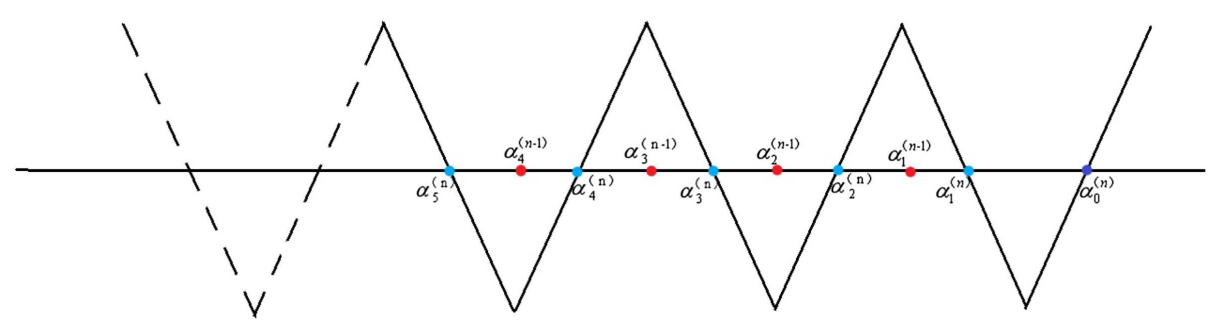

Figure 3. The roots of $\phi_{n}(\xi)=0$.

then

$$
-\left(\lambda \eta_{1}+\mu \eta_{2}\right)-2 \sqrt{\lambda \mu \eta_{1} \eta_{2}} \leq \alpha_{r}^{(n)} \leq-\left(\lambda \eta_{1}+\mu \eta_{2}\right)+2 \sqrt{\lambda \mu \eta_{1} \eta_{2}}(r=1,2, \ldots, n)
$$

and

$$
\left|\alpha_{0}^{(n)}-\alpha_{1}^{(n)}\right| \geq-\left(\lambda \eta_{1}+\mu \eta_{2}\right)+2 \sqrt{\lambda \mu \eta_{1} \eta_{2}} .
$$

Thus 0 is the simple eigenvalue of $A^{(n)}$ for any given $n$.

Moreover, we can deduce that

$$
\phi_{n}(\xi)=\prod_{r=0}^{n}\left(\xi-\alpha_{r}^{(n)}\right)
$$

where $0>\alpha_{1}^{(n)}>\alpha_{2}^{(n)}>\cdots>\alpha_{n}^{(n)}$ and

$$
0=\alpha_{0}^{(n)}=\alpha_{0}^{(n-1)}>\alpha_{1}^{(n)}>\alpha_{1}^{(n-1)}>\cdots>\alpha_{n-1}^{(n)}>\alpha_{n-1}^{(n-1)}>\alpha_{n}^{(n)} .
$$

\subsection{The explicit transient solution}

In order to obtain the explicit transient solution of the asymptotic system (3.1), we need to calculate $\exp \left(t A^{(n)}\right)$. The exponential of a matrix can be calculated in a variety of ways [26], mainly including Taylor series, Padé approximation, ordinary differential equation method, matrix decomposition method. However, Taylor series and Padé approximation are only suitable for smaller matrices. The primary disadvantage of ordinary differential equation method is a relatively high cost in computer time. The matrix decomposition method is especially used for some matrices with special structures. From the properties of eigenvalues of operator $A^{(n)}$ in Theorem 3.1, we use the eigenvector decomposition in matrix decomposition method to solve $\exp \left(t A^{(n)}\right)$.

For convenience, let $\exp \left(t A^{(n)}\right)=\left\{C_{i j}(t)\right\}_{0 \leq i, j \leq n}$. To compute $\left\{C_{i j}(t)\right\}_{0 \leq i, j \leq n}$, we construct non-zero vectors

$$
x_{r}^{(n)}=\left(x_{r 0}^{(n)}, x_{r 1}^{(n)}, x_{r 2}^{(n)}, \ldots, x_{r n}^{(n)}\right), \quad y_{r}^{(n)}=\left(y_{r 0}^{(n)}, y_{r 1}^{(n)}, y_{r 2}^{(n)}, \ldots, y_{r n}^{(n)}\right)
$$

satisfying

$$
\left\{\begin{array}{l}
x_{r}^{(n)}\left(\alpha_{r}^{(n)} I^{(n)}-A^{(n)}\right)=0 \\
\left(\alpha_{r}^{(n)} I^{(n)}-A^{(n)}\right)\left(y_{r}^{(n)}\right)^{T}=0
\end{array}\right.
$$

where $r=0,1,2, \ldots, n$. 
After some elementary calculations, we derive that if $r=0, \alpha_{r}^{(n)}=0$,

$$
\begin{aligned}
x_{0}^{(n)} & =(1,1,1, \ldots, 1) \\
y_{0}^{(n)} & =\left(1, \frac{\lambda \eta_{1}}{\mu \eta_{2}},\left(\frac{\lambda \eta_{1}}{\mu \eta_{2}}\right)^{2}, \ldots,\left(\frac{\lambda \eta_{1}}{\mu \eta_{2}}\right)^{n}\right) \\
\theta_{0}^{(n)} & =x_{0}^{(n)}\left(y_{0}^{(n)}\right)^{T}=\sum_{v=0}^{n}\left(\frac{\lambda \eta_{1}}{\mu \eta_{2}}\right)^{v}=\frac{1-\left(\frac{\lambda \eta_{1}}{\mu \eta_{2}}\right)^{n+1}}{1-\frac{\lambda \eta_{1}}{\mu \eta_{2}}} \\
d_{0}^{(n)}(i, j) & =\frac{\left(y_{0}^{(n)}\right)^{T} x_{0}^{(n)}}{\theta_{0}^{(n)}}=\frac{\left(\mu \eta_{2}-\lambda \eta_{1}\right)\left(\frac{\lambda \eta_{1}}{\mu \eta_{2}}\right)^{i}}{\mu \eta_{2}\left(1-\left(\frac{\lambda \eta_{1}}{\mu \eta_{2}}\right)^{n+1}\right)}
\end{aligned}
$$

and if $r=1,2,3, \ldots, n$,

$$
\begin{aligned}
& x_{r}^{(n)}=\left(1, \frac{1}{\lambda \eta_{1}} \psi_{0}\left(\alpha_{r}^{(n)}\right), \frac{1}{\left(\lambda \eta_{1}\right)^{2}} \psi_{1}\left(\alpha_{r}^{(n)}\right), \ldots, \frac{1}{\left(\lambda \eta_{1}\right)^{n}} \psi_{n}\left(\alpha_{r}^{(n)}\right)\right) \\
& y_{r}^{(n)}=\left(1, \frac{1}{\mu \eta_{2}} \psi_{0}\left(\alpha_{r}^{(n)}\right), \frac{1}{\left(\mu \eta_{2}\right)^{2}} \psi_{1}\left(\alpha_{r}^{(n)}\right), \ldots, \frac{1}{\left(\mu \eta_{2}\right)^{n}} \psi_{n}\left(\alpha_{r}^{(n)}\right)\right)
\end{aligned}
$$

where

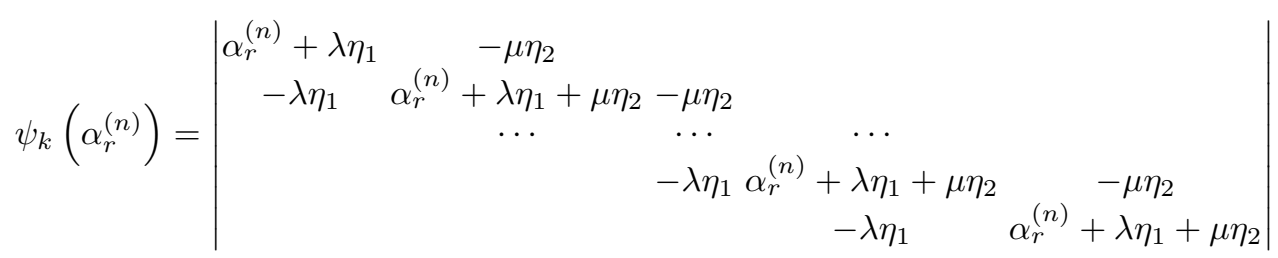

is a matrix with dimensions $(k+1) \times(k+1), k=0,1,2, \ldots, n$ and satisfies the recurrence relation

$$
\psi_{k}\left(\alpha_{r}^{(n)}\right)-\left(\alpha_{r}^{(n)}+\lambda \eta_{1}+\mu \eta_{2}\right) \phi_{k-1}\left(\alpha_{r}^{(n)}\right)+\lambda \mu \eta_{1} \eta_{2} \phi_{k-2}\left(\alpha_{r}^{(n)}\right)=0
$$

which is valid for $k \geq 1$ if we define

$$
\psi_{-1}\left(\alpha_{r}^{(n)}\right)=1, \quad \psi_{0}\left(\alpha_{r}^{(n)}\right)=\alpha_{r}^{(n)}+\lambda \eta_{1} .
$$

Let

$$
\begin{aligned}
\theta_{r}^{(n)} & =x_{r}^{(n)}\left(y_{r}^{(n)}\right)^{T} \\
& =1+\sum_{v=1}^{n} \frac{1}{\left(\lambda \mu \eta_{1} \eta_{2}\right)^{v}}\left\{\psi_{v-1}\left(\alpha_{r}^{(n)}\right)\right\}^{2} \\
& =\sum_{v=0}^{n} \frac{1}{\left(\lambda \mu \eta_{1} \eta_{2}\right)^{v}}\left\{\psi_{v-1}\left(\alpha_{r}^{(n)}\right)\right\}^{2} .
\end{aligned}
$$

The idempotents belonging to $A^{(n)}$ are described by

$$
J_{r}=\frac{\left(y_{r}^{(n)}\right)^{T} x_{r}^{(n)}}{\theta_{r}^{(n)}}=\frac{\left(y_{r}^{(n)}\right)^{T} x_{r}^{(n)}}{x_{r}^{(n)}\left(y_{r}^{(n)}\right)^{T}} .
$$


The matrices (3.12) have the property that

$$
J_{r}^{2}=J_{r}, J_{r} J_{s}=0(r \neq s) .
$$

Then the element in row $i$ and column $j$ of $J_{r}$ is

$$
d_{r}^{(n)}(i, j)=\frac{\left(\frac{1}{\mu \eta_{2}}\right)^{i}\left(\frac{1}{\lambda \eta_{1}}\right)^{j} \psi_{i-1}\left(\alpha_{r}^{(n)}\right) \psi_{j-1}\left(\alpha_{r}^{(n)}\right)}{\theta_{r}^{(n)}}(0 \leq i, j \leq n) .
$$

Since $A^{(n)}$ is a $(n+1) \times(n+1)$ matrix with distinct eigenvalues, then with the spectral resolution [5,20], we deduce

$$
\begin{aligned}
C_{i j}(t) & =\sum_{r=0}^{n} \exp \left(t \alpha_{r}^{(n)}\right) d_{r}^{(n)}(i, j) \\
= & \exp \left(t \alpha_{0}^{(n)}\right) d_{0}^{(n)}(i, j)+\sum_{r=1}^{n} \exp \left(t \alpha_{r}^{(n)}\right) d_{r}^{(n)}(i, j) \\
= & d_{0}^{(n)}(i, j)+\sum_{r=1}^{n} \frac{\exp \left(t \alpha_{r}^{(n)}\right)\left(\frac{1}{\mu \eta_{2}}\right)^{i}\left(\frac{1}{\lambda \eta_{1}}\right)^{j} \psi_{i-1}\left(\alpha_{r}^{(n)}\right) \psi_{j-1}\left(\alpha_{r}^{(n)}\right)}{\theta_{r}^{(n)}} \\
= & \frac{\left(\mu \eta_{2}-\lambda \eta_{1}\right)\left(\frac{\lambda \eta_{1}}{\mu \eta_{2}}\right)^{i}}{\mu \eta_{2}\left(1-\left(\frac{\lambda \eta_{1}}{\mu \eta_{2}}\right)^{n+1}\right)}+\sum_{r=1}^{n} \frac{\exp \left(t \alpha_{r}^{(n)}\right)\left(\frac{1}{\mu \eta_{2}}\right)^{i}\left(\frac{1}{\lambda \eta_{1}}\right)^{j} \psi_{i-1}\left(\alpha_{r}^{(n)}\right) \psi_{j-1}\left(\alpha_{r}^{(n)}\right)}{\theta_{r}^{(n)}} .
\end{aligned}
$$

According to (3.3), we can derive the explicit transient solution of the asymptotic system

$$
\vec{p}^{(n)}(t)=\exp \left(t A^{(n)}\right) \vec{p}^{(n)}(0)=\left(p_{0}^{(n)}(t), p_{1}^{(n)}(t), p_{2}^{(n)}(t), \ldots, p_{n}^{(n)}(t)\right)^{T},
$$

where

$$
\begin{aligned}
p_{i}^{(n)}(t) & =\frac{\left(\mu \eta_{2}-\lambda \eta_{1}\right)\left(\frac{\lambda \eta_{1}}{\mu \eta_{2}}\right)^{i}}{\mu \eta_{2}\left(1-\left(\frac{\lambda \eta_{1}}{\mu \eta_{2}}\right)^{n+1}\right)}+\sum_{r=1}^{n} \frac{\exp \left(t \alpha_{r}^{(n)}\right)\left(\frac{1}{\mu \eta_{2}}\right)^{i} \psi_{i-1}\left(\alpha_{r}^{(n)}\right)}{\theta_{r}^{(n)}} \\
= & \frac{\left(\mu \eta_{2}-\lambda \eta_{1}\right)\left(\frac{\lambda \eta_{1}}{\mu \eta_{2}}\right)^{i}}{\mu \eta_{2}\left(1-\left(\frac{\lambda \eta_{1}}{\mu \eta_{2}}\right)^{n+1}\right)}+\sum_{r=1}^{n} \frac{\exp \left(t \alpha_{r}^{(n)}\right)\left(\frac{1}{\mu \eta_{2}}\right)^{i} \psi_{i-1}\left(\alpha_{r}^{(n)}\right)}{\sum_{v=0}^{n} \frac{1}{\left(\lambda \mu \eta_{1} \eta_{2}\right)^{v}}\left\{\psi_{v-1}\left(\alpha_{r}^{(n)}\right)\right\}^{2}} .
\end{aligned}
$$

\section{TRANSiEnT SOLUtion ANALYSis}

In this section, we use the Trotter-Kato theorem, which is flexible enough to cover various approximation schemes for infinite dimensional systems, to derive transient solution for the CIMS (2.4).

\subsection{The Trotter-Kato theorem}

Before we present the Trotter-Kato theorem, we introduce the following concepts: $A \in G(M, \omega, X)$, $M \geq 1, \omega \in \mathbb{R}$, means that $A$ is the infinitesimal generator of a $C_{0}$-semigroup $T(t), t \geq 0$, satisfying $\|T(t)\| \leq M \cdot \exp (\omega t), t \geq 0 ; \rho(A)$ denote the resolvent set of $A$. 
Theorem 4.1 (Trotter-Kato Theorem [27]). Let $X$ and $X_{n}$ be Banach spaces with norm $\|\cdot\|,\|\cdot\|_{n}, n=1,2, \ldots$, respectively, assume that $A \in G(M, \omega, X), A^{(n)} \in G\left(M, \omega, X_{n}\right), M \geq 1, \omega \in \mathbb{R}$ and for each $n=1,2, \ldots$, there exist bounded linear operators $P_{n}: X \rightarrow X_{n}$ and $E_{n}: X_{n} \rightarrow X$ satisfying:

$\left(a_{1}\right)\left\|P_{n}\right\| \leqslant M_{1},\left\|E_{n}\right\| \leqslant M_{2}$, where $M_{1}$ and $M_{2}$ are independent of $n$.

$\left(a_{2}\right)\left\|E_{n} P_{n} x-x\right\| \rightarrow 0, n \rightarrow \infty$, for all $x \in X$.

(a) $P_{n} E_{n}=I_{n}$, where $I_{n}$ is the identity operator on $X_{n}$.

Let $T(t)$ and $T^{(n)}(t)$ be the semigroups generated by $A$ and $A^{(n)}$ on $X$ and $X_{n}$, respectively. Then the following statements are equivalent:

$\left(A_{1}\right)$ There exists a $\lambda_{0} \in \rho(A) \cap \bigcap_{n=1}^{\infty} \rho\left(A^{(n)}\right)$ such that, for all $x \in X$,

$$
\left\|E_{n}\left(\lambda_{0} I_{n}-A^{(n)}\right)^{-1} P_{n} x-\left(\lambda_{0} I-A\right)^{-1} x\right\| \rightarrow 0, \quad n \rightarrow \infty .
$$

$\left(A_{2}\right)$ For every $x \in X$ and $t \geq 0$,

$$
\left\|E_{n} T^{(n)}(t) P_{n} x-T(t) x\right\| \rightarrow 0, \quad n \rightarrow \infty
$$

uniformly on bounded t-intervals.

In order to apply the Trotter-Kato theorem, it is difficult to verify the resolvent convergence $\left(A_{1}\right)$. To overcome such difficulty, Ito and Kapple [17] indicated that $\left(A_{1}\right)$ can be proved by the following theorem.

Theorem 4.2. Let the assumptions of Theorem 4.1 be satisfied. Then statement $\left(A_{1}\right)$ of Theorem 4.1 is equivalent to $\left(a_{2}\right)$ and the following two statements:

$\left(C_{1}\right)$ There exists a subset $D \subset D(A)$ such that $\bar{D}=X$ and $\overline{\left(\lambda_{0} I-A\right) D}=X$ for a $\lambda_{0}>\omega$.

$\left(C_{2}\right)$ For all $x \in D$ there exist a sequence $\left(\bar{x}_{n}\right)_{n \in N}$ with $\left(\bar{x}_{n}\right)_{n \in N} \in D\left(A^{(n)}\right)$ such that

$$
\lim _{n \rightarrow \infty} E_{n} \bar{x}_{n}=x
$$

and

$$
\lim _{n \rightarrow \infty} E_{n} A^{(n)} \bar{x}_{n}=A x .
$$

The proofs of Theorems 4.1 and 4.2 are shown in [39] and [17] respectively. In view of the Trotter-Kato theorem, the convergence (in an appropriate sense) of the resolvent is equivalent to the convergence of the corresponding semigroup.

\subsection{Transient solution of the CIMS}

The equation (3.14) will be used to construct solutions $p_{i}(t)(i=0,1,2, \ldots)$ of the CIMS (2.4) based on the limiting process, which just reflects the convergence of the semigroups of the Trotter-Kato theorem. So for fixed $t_{0}>0$, we can obtain the transient solution of the CIMS in $t$-intervals $\left[0, t_{0}\right]$ by equation $(3.14)$.

Since the eigenvalues of the matrix $A^{(n)}$ are of the form

$$
\alpha_{0}^{(n)}=0, \alpha_{r}^{(n)}=-\left(\lambda \eta_{1}+\mu \eta_{2}\right)+2 \sqrt{\lambda \mu \eta_{1} \eta_{2}} \cos \omega_{r}^{(n)},
$$

where $\omega_{r}^{(n)}=\frac{r \pi}{n+1}(r=1,2, \ldots, n)$.

Put $\alpha_{r}^{(n)}$ into $\psi_{k}\left(\alpha_{r}^{(n)}\right),(k=1,2,3, \ldots, n)$, we get

$$
\psi_{k}\left(\alpha_{r}^{(n)}\right)=\frac{\left(\sqrt{\lambda \mu \eta_{1} \eta_{2}}\right)^{k}}{\sin \omega_{r}^{(n)}}\left[\sqrt{\lambda \mu \eta_{1} \eta_{2}} \sin \left((k+2) \omega_{r}^{(n)}\right)-\mu \eta_{2} \sin \left((k+1) \omega_{r}^{(n)}\right)\right] .
$$


According to (3.11), we have

$$
\begin{aligned}
\theta_{0}^{(n)} & =\frac{1-\left(\frac{\lambda \eta_{1}}{\mu \eta_{2}}\right)^{n+1}}{1-\frac{\lambda \eta_{1}}{\mu \eta_{2}}}, \\
\theta_{r}^{(n)} & =-\frac{1}{\lambda \eta_{1}} \frac{\alpha_{r}^{(n)}(n+1)}{2 \sin ^{2} \omega_{r}^{(n)}}, \quad(r=1,2, \ldots, n) .
\end{aligned}
$$

Let $\Delta \omega^{(n)}=\omega_{r+1}^{(n)}-\omega_{r}^{(n)}=\frac{\pi}{n+1}$, then we get the following results

$$
\frac{1}{\theta_{r}^{(n)}}=\lambda \eta_{1} \frac{2 \sin ^{2} \omega_{r}^{(n)}}{\pi\left(-\alpha_{r}^{(n)}\right)} \Delta \omega^{(n)}, \quad(r=1,2,3, \ldots)
$$

For the operator $A^{(n)}$, we define $\sigma^{(n)}(x)$ as follows: $\sigma^{(n)}(x)$ is to be a non-decreasing step function, defined for $-\infty<x<\infty$, with discontinuities $\frac{1}{\theta^{(n)}}$ at $x=\alpha_{r}^{(n)}(r=0,1, \ldots, n)$ and $\sigma^{(n)}(0)=0$.

Explicitly, $\sigma^{(n)}(x)$ is given by

$$
\sigma^{(n)}(x)= \begin{cases}0, & x \geq \alpha_{0}^{(n)} \\ -\left(\frac{1}{\theta_{0}^{(n)}}+\frac{1}{\theta_{1}^{(n)}}+\cdots+\frac{1}{\theta_{r}^{(n)}}\right), & \alpha_{r+1}^{(n)} \leq x<\alpha_{r}^{(n)}, \quad(r=0,1, \ldots, n-1) \\ -\left(\frac{1}{\theta_{0}^{(n)}}+\frac{1}{\theta_{1}^{(n)}}+\cdots+\frac{1}{\theta_{n}^{(n)}}\right), & x<\alpha_{n}^{(n)} .\end{cases}
$$

As $n \rightarrow \infty$, let

$$
\omega=\lim _{n \rightarrow \infty} \omega_{r}^{(n)}
$$

we have

$$
\begin{gathered}
\sigma_{0}=\lim _{n \rightarrow \infty} \frac{1}{\theta_{0}^{(n)}}= \begin{cases}0, & \lambda \eta_{1} \geq \mu \eta_{2} \\
\frac{\mu \eta_{2}-\lambda \eta_{1}}{\mu \eta_{2}}, & \lambda \eta_{1}<\mu \eta_{2}\end{cases} \\
\mathrm{d} \sigma(x)=\lambda \eta_{1} \frac{2 \sin ^{2} \omega}{\pi(-x)} \mathrm{d} \omega, \quad(0 \leq \omega \leq \pi)
\end{gathered}
$$

and the second part of (3.14) can be expressed as an integral, that is

$$
\sum_{r=1}^{\infty} \frac{\exp \left(t \alpha_{r}^{(n)}\right)\left(\frac{1}{\mu \eta_{2}}\right)^{i} \psi_{i-1}\left(\alpha_{r}^{(n)}\right)}{\theta_{r}^{(n)}}=\left(\frac{1}{\mu \eta_{2}}\right)^{i} \int_{-\infty}^{0} \psi_{i-1}(x) \exp (t x) \mathrm{d} \sigma(x), \quad(i=0,1,2, \ldots, n) .
$$

So the transient solution of the CIMS $\left(t \in\left[0, t_{0}\right]\right)$ is $\vec{p}(t)=\left(p_{0}(t), p_{1}(t), p_{2}(t), \ldots\right)^{T}$, where

$$
\begin{aligned}
p_{i}(t) & =\lim _{n \rightarrow \infty} p_{i}^{(n)}(t) \\
& =\frac{\mu \eta_{2}-\lambda \eta_{1}}{\mu \eta_{2}}\left(\frac{\lambda \eta_{1}}{\mu \eta_{2}}\right)^{i}+\left(\frac{1}{\mu \eta_{2}}\right)^{i} \int_{-\infty}^{0} \psi_{i-1}(x) \exp (t x) \mathrm{d} \sigma(x) \\
& =\frac{\mu \eta_{2}-\lambda \eta_{1}}{\mu \eta_{2}}\left(\frac{\lambda \eta_{1}}{\mu \eta_{2}}\right)^{i}-\frac{2}{\pi} \exp \left(-\left(\lambda \eta_{1}+\mu \eta_{2}\right) t\right)\left(\frac{\lambda \eta_{1}}{\mu \eta_{2}}\right)^{\frac{i}{2}} \cdot U_{1}, \quad(i=0,1,2, \ldots, n),
\end{aligned}
$$

in which

$$
U_{1}=\int_{0}^{\pi} \frac{\lambda \eta_{1}(\sin \omega)(\sin (i+1) \omega)-\sqrt{\lambda \mu \eta_{1} \eta_{2}}(\sin \omega)(\sin i \omega)}{-\left(\lambda \eta_{1}+\mu \eta_{2}\right)+2 \sqrt{\lambda \mu \eta_{1} \eta_{2}} \cos \omega} \exp \left(2 \sqrt{\lambda \mu \eta_{1} \eta_{2}} t \cos \omega\right) \mathrm{d} \omega
$$


Moreover, if we let $t$ tends to infinity in (4.4), we have,

$$
\vec{p}^{\star}=\left(p_{0}^{\star}, p_{1}^{\star}, p_{2}^{\star}, \ldots\right)^{T},
$$

where

$$
p_{i}^{\star}=\lim _{t \rightarrow \infty} p_{i}(t)=\frac{\mu \eta_{2}-\lambda \eta_{1}}{\mu \eta_{2}}\left(\frac{\lambda \eta_{1}}{\mu \eta_{2}}\right)^{i}
$$

which just satisfies equation (2.5) and

$$
\lim _{n \rightarrow \infty} p_{i}^{(n)^{\star}}=p_{i}^{\star}
$$

where $p_{i}^{(n)^{\star}}=\frac{\left(\mu \eta_{2}-\lambda \eta_{1}\right)\left(\frac{\lambda \eta_{1}}{\mu \eta_{2}}\right)^{i}}{\mu \eta_{2}\left(1-\left(\frac{\lambda \eta_{1}}{\mu \eta_{2}}\right)^{n+1}\right)},(i=0,1,2, \ldots, n)$ is derived from equation (3.14) by letting $t \rightarrow \infty$ in $p_{i}^{(n)}(t)$.

Therefore, as $t$ tends to infinity, the Trotter-Kato theorem still applies to the transient solution of the CIMS.

\subsection{Convergence analysis}

Equation (4.1) yields that

$$
-\left(\lambda \eta_{1}+\mu \eta_{2}\right)-2 \sqrt{\lambda \mu \eta_{1} \eta_{2}} \leq \alpha_{r}^{(n)} \leq-\left(\lambda \eta_{1}+\mu \eta_{2}\right)+2 \sqrt{\lambda \mu \eta_{1} \eta_{2}} .
$$

The spectral interval in (4.6) is independent of $n$. As a result, the transient solution of the CIMS converges exponentially to its steady-state solution as shown in the following Theorem.

Theorem 4.3. The transient solution of the system (2.4) converges exponentially to its steady-state solution. That is

$$
\left\|\vec{p}(t)-\vec{p}^{\star}\right\| \leq e^{-\omega t},
$$

where $\omega=\left(\lambda \eta_{1}+\mu \eta_{2}\right)-2 \sqrt{\lambda \mu \eta_{1} \eta_{2}}>0$.

\section{NumERICAL EXAMPLES}

In this section, some numerical examples are presented to illustrate the effectiveness of the proposed approximation method.

First, we will verify that the asymptotic system constructed in this paper is feasible.

To simplicity the simulation and satisfy $\frac{\lambda \eta_{1}}{\mu \eta_{2}}<1$, we assume $\lambda=0.6, \eta_{1}=0.4, \mu=0.8, \eta_{2}=0.6$. By equations (3.14) and (4.4), we can get the explicit transient solution $\vec{p}^{(n)}(t)=\left(p_{0}^{(n)}(t), p_{1}^{(n)}(t), p_{2}^{(n)}(t), \ldots, p_{n}^{(n)}(t)\right)^{T}$ of the asymptotic system and the transient solution $\vec{p}(t)=\left(p_{0}(t), p_{1}(t), p_{2}(t), \ldots\right)^{T}$ of the CIMS, where the transient behaviors of $p_{0}^{(1)}(t), p_{0}^{(3)}(t), p_{0}^{(5)}(t), p_{0}(t)\left(p_{0}^{(\infty)}(t)\right)$ are depicted in Figure 4.

In Figure 4, the explicit transient solution of the asymptotic system tends to the transient solution of the CIMS with $n$ increasing. Figure 5 shows the transient behaviors $p_{i}(t), i=0,1,2,3$ of the CIMS. Notice that with the increase of time $t$, the transient solution of the CIMS tends to the steady-state solution $\left(\frac{1}{2}, \frac{1}{4}, \frac{1}{8}, \frac{1}{16}, \ldots\right)^{T}$ that satisfies equation (4.5), which reflects the validity of the Theorem 4.3. Therefore, the asymptotic system constructed in this paper is feasible.

Second, we will show the effect of the system parameters $\eta_{i}, i=1,2$ on the behavior of the CIMS.

$p_{0}(t)$ denotes the probability that machine 1 is processing a workpiece at time $t$ and there is no workpiece in the buffer or machine 2. With the change of probabilities $\eta_{i}, i=1,2$, the curves of $p_{0}(t)$ are provided in Figures 6 and 7 . It is obvious that $p_{0}(t)$ are decreasing to a steady-state solution, but the steady-state solution will vary with different $\eta_{1}$ and $\eta_{2}$. Moreover, the steady-state solution decreases with the increase of $\eta_{1}$ and increases with the increase of $\eta_{2}$.

For $\lambda=0.6, \mu=0.8, t=100$, Figure 8 is the three-dimensional diagram of $\eta_{1}, \eta_{2}$ and $p_{0}(100)$. From Figure 8 , we can find the relationship between $p_{0}(100)$ and $\eta_{1}, \eta_{2}$, namely $p_{0}(100)$ decreases as $\eta_{1}$ increases while $p_{0}(100)$ 


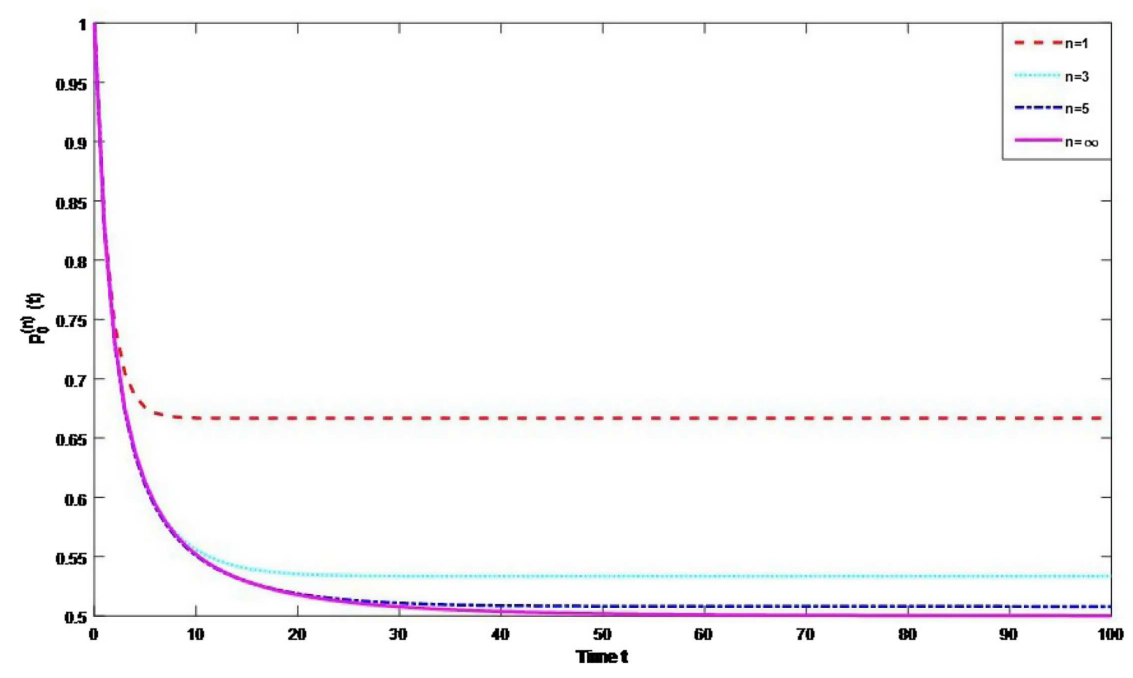

Figure 4. Transient behaviors for the asymptotic system and the CIMS.

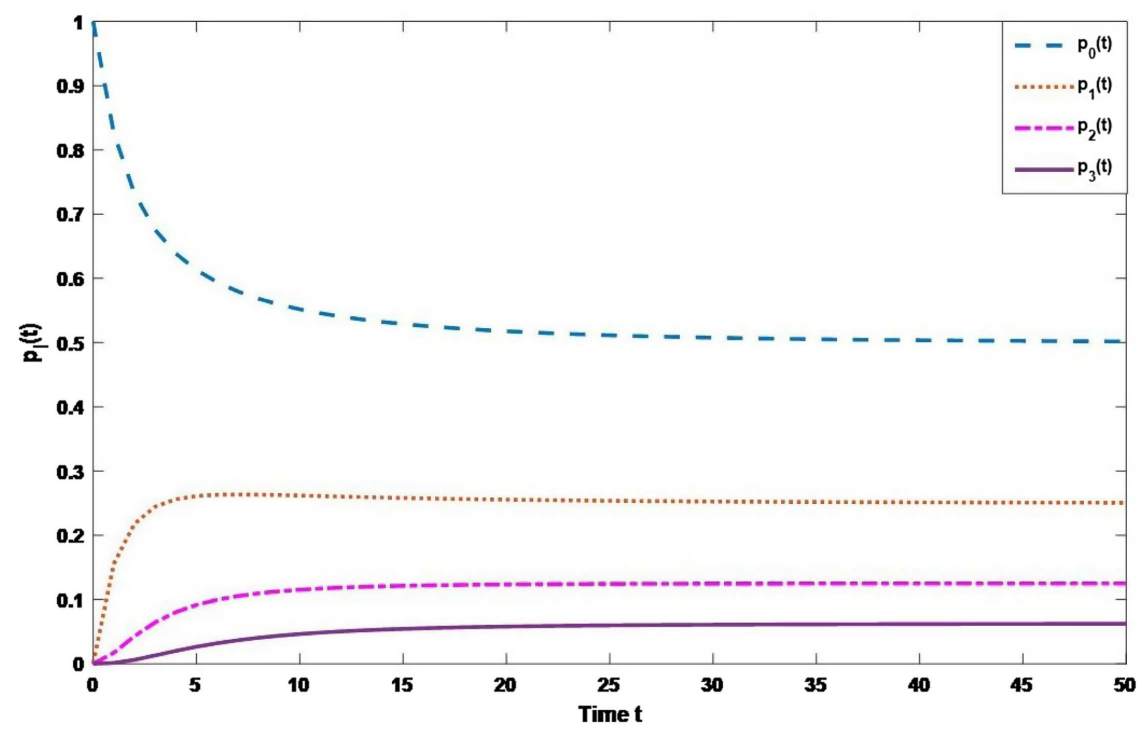

Figure 5. Transient behaviors of the CIMS with $\lambda=0.6, \eta_{1}=0.4, \mu=0.8, \eta_{2}=0.6$.

increases as $\eta_{2}$ increases. We can also deduce that the steady-state solution of $p_{0}(t)$ will decreases with the increase of $\eta_{1}$ and increases with the increase of $\eta_{2}$.

Finally, we will consider the behavior of the CIMS without Bernoulli feedbacks $\left(\eta_{1}=\eta_{2}=1\right)$.

Figure 9 illustrates the transient behaviors $p_{i}(t), i=0,1,2,3$ of the CIMS without Bernoulli feedbacks. We can see that the transient solution of the CIMS without Bernoulli feedbacks tends to its steady-state solution $\left(\frac{1}{4}, \frac{3}{16}, \frac{9}{64}, \frac{27}{256}, \ldots\right)^{T}$ that satisfies equation $(4.5)$. 


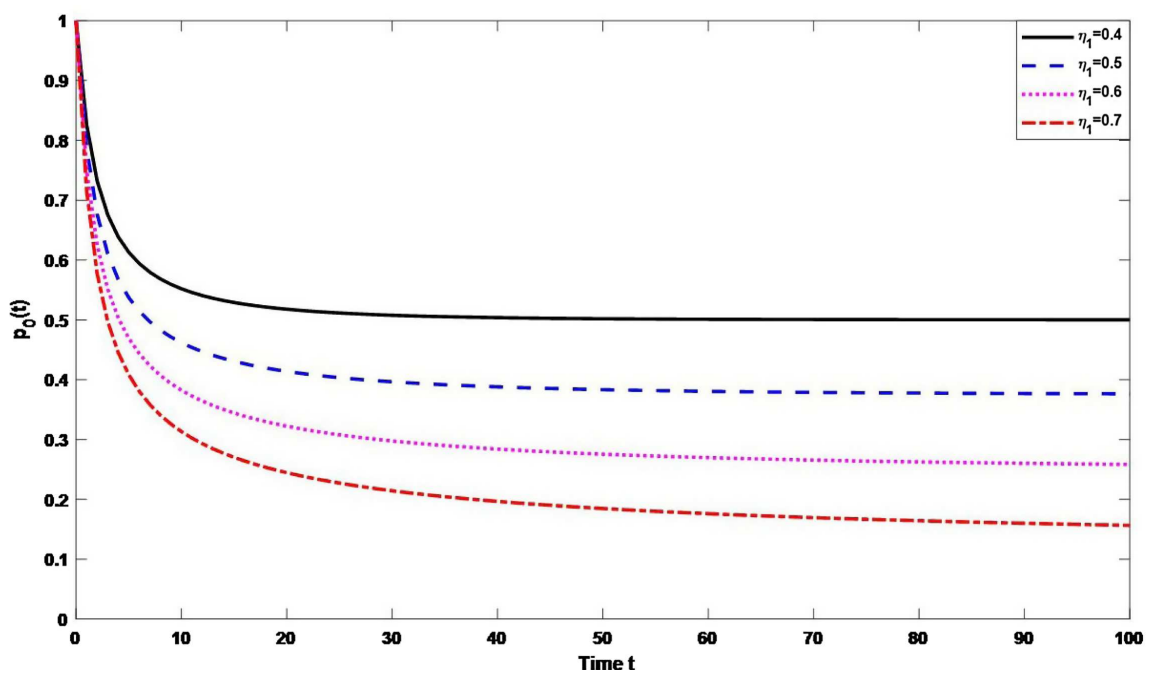

FiguRE 6. $p_{0}(t)$ with the change of $\eta_{1}$.

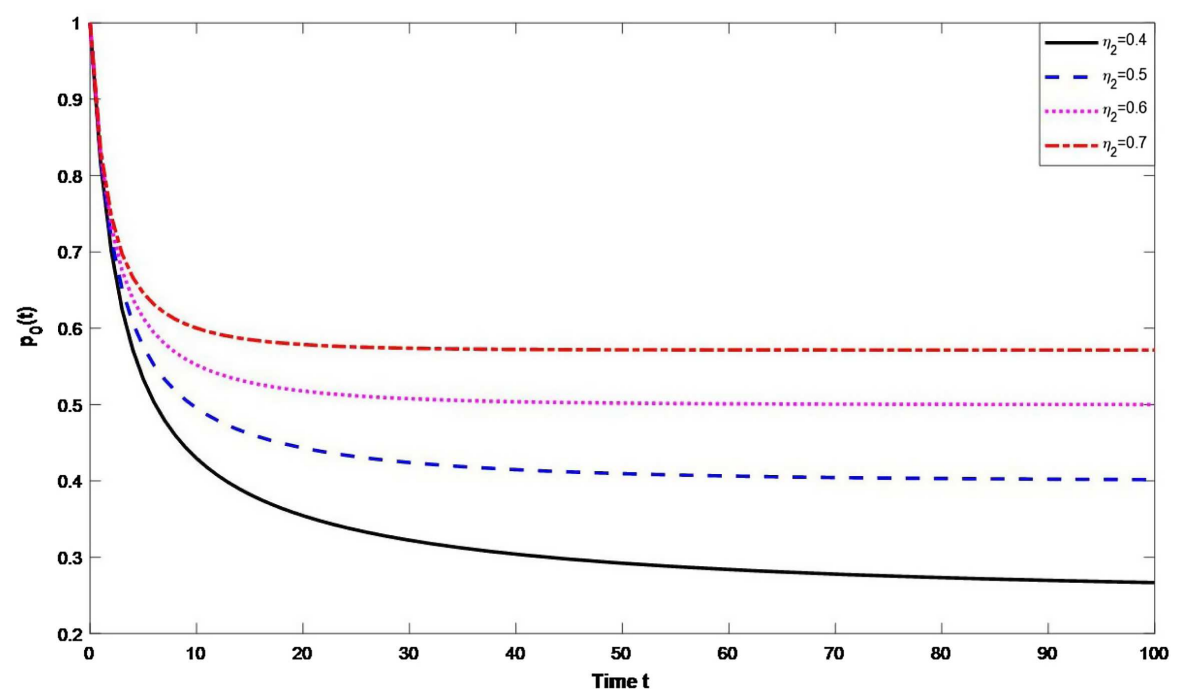

Figure 7. $p_{0}(t)$ with the change of $\eta_{2}$.

\section{Conclusion}

Transient analysis, including the transient solution and the convergence rate of the transient solution, is of great value in understanding system performance. This paper discussed the transient behavior of a typical single server queueing system with infinite buffer, namely CIMS, by spectral distribution method. We have derived the transient solution of the CIMS, which is the limitation of the explicit transient solution of the constructed asymptotic system with the method of difference scheme by Trotter-Kato theorem. Furthermore, we have proved that in the CIMS the transient solution converged to the steady-state solution exponentially with exponential 


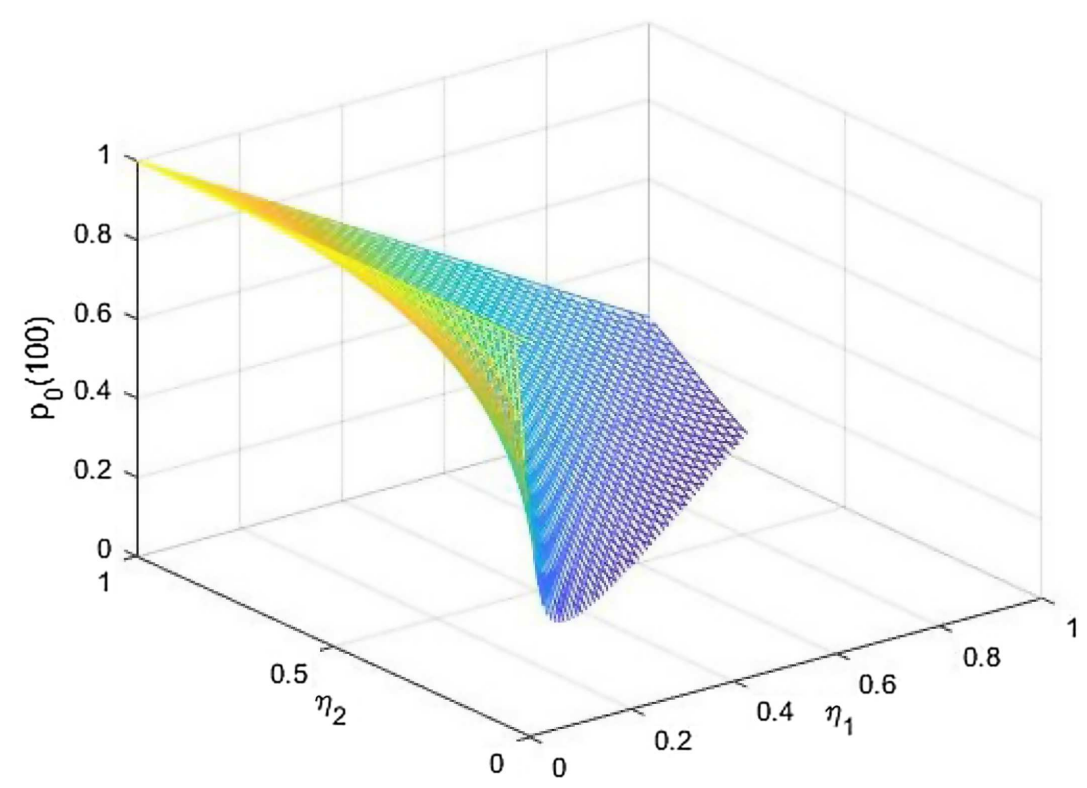

Figure 8. Three dimensional diagram of $\eta_{1}, \eta_{2}, p_{0}(100)$.

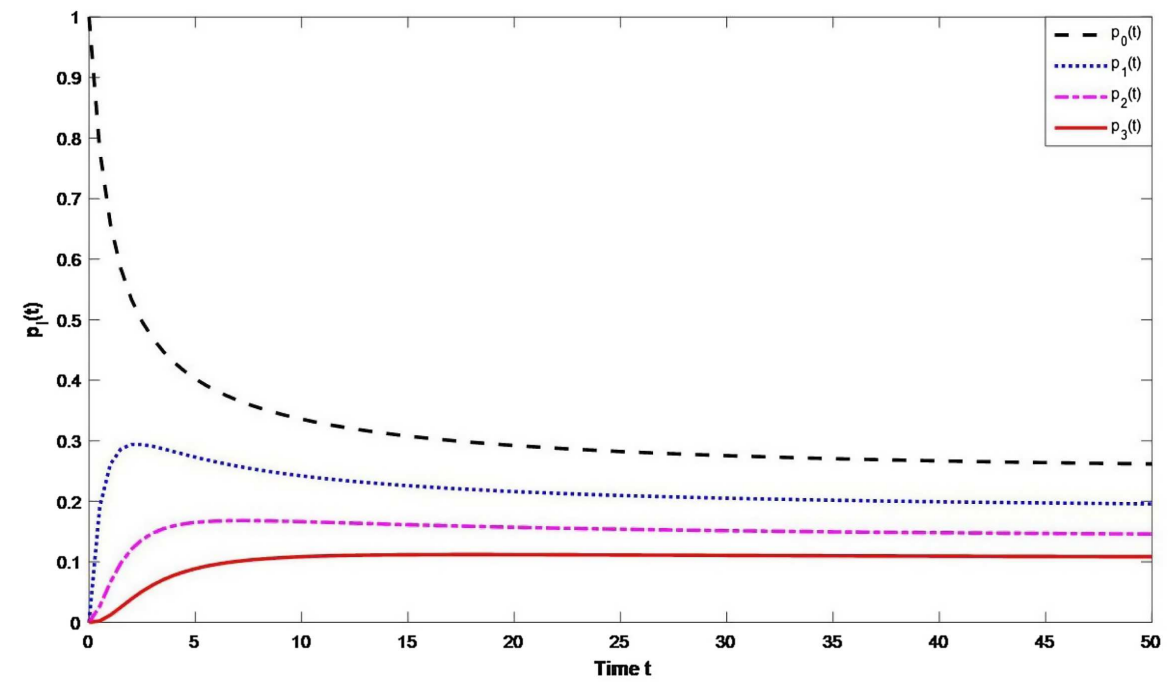

Figure 9. Transient solution of the CIMS with $\lambda=0.6, \mu=0.8, \eta_{1}=\eta_{2}=1$.

bound $-\omega=-\left(\lambda \eta_{1}+\mu \eta_{2}\right)+2 \sqrt{\lambda \mu \eta_{1} \eta_{2}}$. Numerical examples are also given to illustrate the validity of the proposed approximation in the paper.

Acknowledgements. The authors gratefully acknowledge the contribution of NSAF (U1430125) and thank the editor and anonymous reviewers for their valuable and constructive comments that have improved this paper. 


\section{REFERENCES}

[1] F. Afroun, D. Aissani, D. Hamadouche and M. Boualem, Q-matrix method for the analysis and performance evaluation of unreliable M/M/1/N queueing model. Math. Methods Appl. Sci. 18 (2018) 9152-9163.

[2] N. Barbot and B. Sericola, Stationary solution to the fluid queue fed by an $M / M / 1$ queue. J. Appl. Probab. 39 (2002) $359-369$.

[3] J. Braband, Waiting time distributions for closed $M / M / N$ processor sharing queues. Queueing Syst. 19 (1995) 331-344.

[4] V.V. Chaplygin, A multiserver queueing system with a interruptable semi-Markovian input flow of customers and customer removal from an infinite buffer. J. Commun. Technol. Electron. 55 (2010) 1491-1498.

[5] F. Chatelin, Spectral Approximation of Linear Operators. Tianjin University Press (1987).

[6] M.L. Chaudhry, A.D. Banik, A. Pacheco and S. Ghosh, A simple analysis of system characteristics in the batch service queue

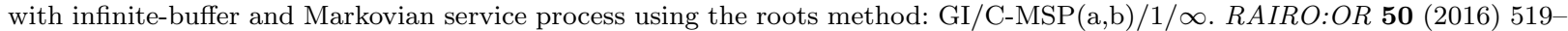
551.

[7] A. Chydzinski, M. Barczyk and D. Samociuk, The single-server queue with the dropping function and infinite buffer. Math. Prob. Eng. 2018 (2018) 1-12.

[8] J.W. Cohen, Superimposed renewal process and storage with gradual input. Stoch. Process. App. 2 (1974) 31-58.

[9] F.R.B. Cruz, A.R. Duarte and G.L. Souza, Multi-objective performance improvements of general finite single-server queueing networks. J. Heuristics 24 (2018) 757-781.

[10] P. Fleming and B. Simon, Interpolation approximations of sojourn time distribution. Oper. Res. 39 (1991) $251-260$.

[11] L.C. Gerardo, F. Ricardo and P. Elias, On dynamical behaviour of two-dimensional biological reactors. Int. J. Syst. Sci. 43 (2012) 526-534.

[12] A.P. Ghosh and A.P. Park, Optimal buffer size and dynamic rate control for a queueing system with impatient customers in heavy traffic. Stoch. Process. App. 120 (2010) 2103-2141.

[13] N.I. Golovko, V.O. Karetnik and O.V. Peleshok, Queuing system with infinite buffer and stepwise inflow intensity. Autom. Remote Control 70 (2009) 1662-1682.

[14] G. Gupur and X.-Z. Li, A note on the $M / M / 1$ queueing model described by ordinary differential equation. Acta Anal. Funct. Appl. 1 (1999) 69-74.

[15] G. Gupur, X.-Z. Li and G.-T. Zhu, Functional Analysis Method in Queueing Theory. Research Information Ltd, Hertfordshire (2001).

[16] F.L. Huang, Strong asymptotic stability of linear dynamical systems in Banach spaces. J. Differ. Equ. 104 (1993) $307-324$.

[17] K. Ito and F. Kappel, The Trotter-Kato theorem and approximation of PDEs. Math. Comput. 67 (1998) 21-44.

[18] W.M. Kempa and M. Kobielnik, Transient solution for the queue-size distribution in a finite-buffer model with general independent input stream and single working vacation policy. Appl. Math. Model. 59 (2018) 614-628.

[19] A. Krinik, Taylor series solution of the $M / M / 1$ queueing system. J. Comput. Appl. Math. 44 (1992) 371-380.

[20] W. Ledermann and G.E.H. Reuter, Spectral theory for the differential equations of simple birth and death processes. Math. Phys. Sci. 246 (1954) 1934-1990.

[21] P. Leguesdron, J. Pellaumail, G. Rubino and B. Sericola, Transient analysis of the $M / M / 1$ queue. Adv. Appl. Probab. 25 (1993) $702-713$.

[22] R.R. Levary, Computer integrated manufacturing: a complex information system. Prod. Plan. Control 7 (1996) 184-189.

[23] C.-H. Liu, Queuing Theory. Beijing University of Posts and Telecommunications (2009).

[24] Y.M. Liu, X.F. Li, W.X. Wang and R.Y. Song, Dynamical solution and asymptotic stability of the $M / M / n$ model with variable import rate. Math. Appl. 22 (2009) 705-710.

[25] D. Mitra, Stochastic theory of a fluid model of producers and consumers coupled by a buffer. Adv. Appl. Probab. 20 (1988) $646-676$.

[26] C. Moler and C. V. Loan, Nineteen dubious ways to compute the exponential of a matrix. Soc. Ind. Appl. Math. 20 (1978) 801-836.

[27] A. Pazy, Semigroups of Linear Operators and Applications to Partial Differential Equations. In: Vol.44 of Applied Mathematical Sciences. Springer, New York, NY (1983).

[28] S. Pradhan and U.C. Gupta, Analysis of an infinite-buffer batch-size-dependent service queue with Markovian arrival process. Ann. Oper. Res. 277 (2019) 161-196.

[29] S. Pradhan, U.C. Gupta and S.K. Samanta, Analyzing an infinite buffer batch arrival and batch service queue under batchsize-dependent service policy. J. Korean Stat. Soc. 45 (2016) 137-148.

[30] B. Sericola, Transient analysis of stochastic fluid models. Perform. Eval. 32 (1998) 245-263.

[31] S. Sericola, A finite buffer fluid queue driven by a Markovian queue. Queueing Syst. 38 (2001) 213-220.

[32] B. Sericola, P.R. Parthasarathy and K.V. Vijayashree, Exact transient solution of an $M / M / 1$ driven fluid queue. Int. J. Comput. Math. 82 (2005) 659-671.

[33] S. Sharma, R. Kumar and S.I. Ammar, Transient and steady-state analysis of a queuing system having customers' impatience with threshold. RAIRO:OR 53 (2019) 1861-1876.

[34] S.G. Shu, An analysis of the repairable CIMS with buffers and a study of system reliability. Acta Auto. Sin. 18 (1992) 15-22.

[35] B. Tjahjono, C. Esplugues, E. Ares and G. Pelaez, What does industry 4.0 mean to supply chain? Proc. Manuf. 13 (2017) $1175-1182$

[36] S. Vaidya, P. Ambad and S. Bhosle, Industry 4.0 - a glimpse. Proc. Manuf. 20 (2018) 233-238. 
[37] K. Wu and N. Zhao, Analysis of dual tandem queues with a finite buffer capacity and non-overlapping service times and subject to breakdowns. IIE Trans. 47 (2015) 1329-1341.

[38] G.-H. Xu, Random Service System. Science Press, Beijing (1984).

[39] H.B. Xu and W.W. Hu, Analysis and approximation of a reliable model. Appl. Math. Model. 37 (2013) $3777-3788$.

[40] H.Y. Zhang, Q.X. Chen, J.M. Smith, N. Mao, A.L. Yu and Z.T. Li, Performance analysis of open general queuing networks with blocking and feedback. Int. J. Prod. Res. 55 (2017) 5760-5781. 